\title{
"Piler Dirt" Survey for the Sampling and Detection of Potato Cyst Nematodes
}

\author{
Benjamin Mimee, ${ }^{\dagger}$ Nathalie Dauphinais, and Guy Bélair \\ Agriculture and Agri-Food Canada, Saint-Jean-sur-Richelieu Research and Development Centre, 430 boulevard Gouin,
} Saint-Jean-sur-Richelieu, QC, J3B 3E6 Canada

\begin{abstract}
Potato cyst nematodes are a significant threat to potato production worldwide and have important economic impacts due to yield losses but also because of the expenses associated with regulation procedures. In order to reduce the sampling labor, an alternative strategy named the "Piler Dirt" that collects the soil carried with potato tubers during their transfer to storage was proposed. The method showed a better sensitivity than the reference method to detect fields infested with $G$. rostochiensis. The quantification of the number of cysts per kilogram of soil was propor-

treated by diagnostic labs. It was shown that subsampling six aliquots, each equivalent to $5,000 \mathrm{~cm}^{3} /$ ha, from the total quantity of soil generated by the Piler Dirt method, resulted in a probability of $97 \%$ to detect infested fields, $95 \%$ of the time in our dataset. Overall, Piler Dirt appears as a good compromise to reduce labor time and cost without significantly affecting sensitivity. However, it will be challenging to implement because it needs to be done simultaneously with harvest and will require the participation of farmers during a busy period.
\end{abstract} tional between the two methods at low and moderate population densities $\left(R^{2}=0.885\right)$ but no correlations were found at high density. However, the quantity of soil generated by the method was exceedingly large to be
Keywords: detection, Globodera rostochiensis, Piler Dirt, potato cyst nematode, soil sampling.
Potato (Solanum tuberosum L.) is the third most produced food crop after wheat and rice, with an annual production of 380 million tons (FAO 2018). It is an important source of carbohydrates and vitamins, especially in developing countries where the production has significantly increased over the past decade (Zaheer and Akhtar 2016). Among the most important threats to potato production worldwide are the potato cyst nematodes (PCN) Globodera rostochiensis (Wollenweber, 1923: Skarbilovich, 1959), Behrens, and G. pallida (Stone, 1973). If left uncontrolled, they can cause up to $100 \%$ loss in potato yields (Baldwin and Mundo-Ocampo 1991; Brodie et al. 1998). These nematodes have acquired the remarkable ability to survive unsuitable conditions for decades in soil (Phillips 1994) and to hatch only in the presence of a potential host by rapidly modulating gene expression in response to stimuli (Duceppe et al. 2017; Sabeh et al. 2018). Because of this high impact on yield and the difficulties associated with their management, $\mathrm{PCN}$ are subject to strict regulation in many countries (Seehofer 2007). In North America, the discovery of $G$. rostochiensis in Quebec (Sun et al. 2007) and $G$. pallida in Idaho (Hafez et al. 2007) has had a significant impact on the potato industry and international trade. In both cases, these infestations are most probably the result of new introductions from Europe rather than the spread of other North American populations (Boucher et al. 2013; Mimee et al. 2015b). From now on, serious precautions need to be taken in order to contain the spread of these PCN populations to other potato-growing areas. A wide investigation was undertaken by the Canadian Food and Inspection Agency (CFIA) in Canada and the United States Department of Agriculture-Animal

${ }^{\dagger}$ Corresponding author: B. Mimee; benjamin.mimee@ canada.ca

Funding: This work was supported by the Research Partnership Strategy program of the Canadian Food Inspection Agency (CHA-P-1430) and the United States Department of Agriculture through a Coordinated Agricultural Project titled "Risk Assessment and Eradication of Globodera spp. in U.S. Production of Potato," supported by the National Institute for Food and Agriculture (https://www.globodera.org/) award \#2015-69004-23634.

The author(s) declare no conflict of interest

Accepted for publication 20 March 2019.

(C) 2019 Her Majesty the Queen in Right of Canada, as represented by Agriculture and Agri-Food Canada (AAFC). and Plant Health Inspection Service in the United States to determine the extent of the infestation. In 2006, a binational agreement established the guidelines on surveillance and phytosanitary actions for G. rostochiensis and G. pallida (Canada and United States 2014), including mandatory surveillance of seed potato fields.

Although necessary, these field surveys are time consuming and costly. They also require that technicians walk from field to field and bring soil samples back to the diagnostic labs. Together, these movements of soil and people could generate biosecurity concerns. An alternative sampling strategy that collects the soil carried with potato tubers during harvest when they are transferred to storage by producers was proposed. This "Piler Dirt" soil survey could be an interesting way to achieve the required soil sampling effectively and efficiently. Sampling directly into this dirt would eliminate the need to enter the field and considerably reduce the sampling time. Soil collected under conveyors or in potato warehouses was already shown to be a significant source of PCN, contributing to its propagation (Goeminne et al. 2011). Incidentally, the presence of G. pallida in Idaho was discovered during a routine survey of tare dirt at a processing facility (Hafez et al. 2007). A report from Belgium also indicated that sampling the soil falling from the conveyor belt during harvest was promising (Goeminne et al. 2015). The authors showed a good relation between the number of cysts detected using this technique compared with the manual reference method after a crop of susceptible potato. Here, it is proposed to investigate the sensitivity and accuracy of the Piler Dirt survey compared with the official hand-sampling method ("Method B") in quarantine fields growing resistant potato or nonhost plants for at least 8 years. The results from this project will enhance the capacity of the inspection agencies to detect and follow PCN populations while reducing the time, cost, and labor necessary to meet regulatory requirements.

\section{Materials and Methods}

Potato fields. The project took place on three consecutive years starting during fall 2014 in the PCN quarantine area of St-Amable, QC, Canada (CFIA 2006). Samplings were done in commercial potato fields naturally infested by G. rostochiensis (pathotype Ro1) (Mahran et al. 2010) and cropped with resistant potato cultivars carrying the $\mathrm{H} 1$ gene. All of the fields were in potato production during the sampling year and 3 years previously, and were cropped with nonhost plants (corn or soybean) in the remaining years since 2007. Potato crops were grown under normal management practices and top killed after approximately 100 days of growth. Two sampling 
methods (detailed below) were compared: the Piler Dirt, resulting from the movement of potato from a transport truck to a storage facility, and Method B, a manual composite sampling following a 4-by-6-m grid. Method B is the official method recommended by the report "Canada and United States guidelines on surveillance and phytosanitary actions for the PCN G. rostochiensis and G. pallida" (Canada and United States 2014).

In total, 46 strips of about 0.2 ha were tested during the project. They were located in 24 potato fields representing three groups of initial infestation levels: low (0 to 5 cysts/kg of dry soil; 29 strips), moderate ( 5 to 25 cysts $/ \mathrm{kg}$ of dry soil; 9 strips), and high ( $\geq 100$ cysts $/ \mathrm{kg}$ of dry soil; 8 strips). Each strip was considered as an experimental unit and was georeferenced with a global positioning system device in order to sample the same area using both methods. The coordinates were also used to calculate the exact quantity of soil to sample to obtain $5,000 \mathrm{~cm}^{3} /$ ha for Method B in each.

Piler Dirt survey. The Piler Dirt sampling was conducted during harvest, 3 to 4 weeks after potato plants were top killed. Soil collection was achieved using a plastic membrane placed under the bulk box or conveyor belt. All of the soil falling from the equipment while the potato tubers were being unloaded from the transport truck and transferred to the storage boxes was collected on the membrane. The area under the bulk box and the conveyor belt was cleaned between each strip. The total quantity of soil collected on the plastic membrane was transferred into pails, homogenized, and subdivided into subsamples of a volume equal to a Method B sample from the same strip (each one corresponding to $5,000 \mathrm{~cm}^{3} / \mathrm{ha}$ ) and deposited in paper bags.

Hand sampling with Method B. Immediately after harvesting, the Method B was carried out. The hand sampling was done over the entire strips in a regular 4-by-6-m grid pattern. Small aliquots of soil were collected at each intersection of the grid and pooled together to obtain $5,000 \mathrm{~cm}^{3}$ of soil per hectare $\left(2,000 \mathrm{~cm}^{3} / \mathrm{acre}\right)$. Because all strips were rectangular shaped, the hand sampling was done in parallel to the direction of cultivation, according to NPPO guidelines (Canada and United States 2014). Soil samples were taken using a tiny trowel designed according to the specifications of the CFIA for soil sampling. Each aliquot contained about $12 \mathrm{~cm}^{3}$ of soil. Samples were deposited in a pail, homogenized, and distributed in paper bags according to the quantity estimated to obtain $5,000 \mathrm{~cm}^{3} /$ ha for each strip. Three independent samples were taken in each strip by moving the first sampling point by a distance of $2 \mathrm{~m}$ each time. In this manner, Method B was performed three times.

Cyst extraction. Soil samples were allowed to dry in paper bags and shipped to the CFIA Plant Health Laboratory located in Charlottetown, PEI, Canada. Each soil sample was weighed before cyst extraction using the Fenwick can method (Fenwick 1940). Samples were extracted in their entirety and the number of cysts was recorded by visual examination using a stereomicroscope.

Statistical analyses. Analyses of variance were performed using XLSTAT 2015 (Addinsoft, New York, NY, U.S.A.). The probabilities of detection of $G$. rostochiensis with different numbers of Piler

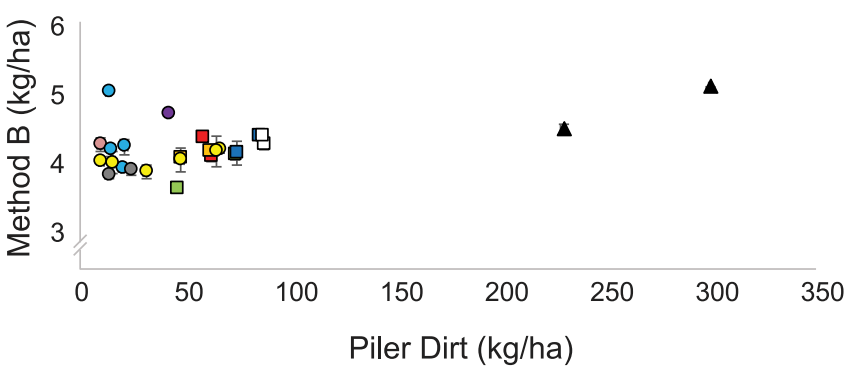

Fig. 1. Weight of soil samples obtained using the Piler Dirt procedure compared with the official standard Method B from the same areas in 2015 and 2016. Error bars are the standard error of three independent samplings using Method B. Plots from a same field (but different strips) are indicated by the same color and fields from the same producer by the same shape.
Dirt subsamples were calculated using the urn model with simultaneous draws, without replacement.

\section{Results}

The quantity of soil obtained using the Piler Dirt sampling procedure was significantly higher and much more variable than the official Method B, with an average of $62.12 \pm 12.89 \mathrm{~kg} / \mathrm{ha}$ ( \pm standard error) compared with $4.28 \pm 0.07 \mathrm{~kg} / \mathrm{ha}$ (Fig. 1). The weight of the Piler Dirt samples also differed significantly between growers and, to a lesser extent, from different samples from the same field (Fig. 1). When compared with Method B, the Piler Dirt method was slightly better at detecting fields infested with G. rostochiensis (although much more soil was processed). Both methods were in agreement in 36 of 46 fields (34 positive and 2 negative) (Fig. 2A). The Piler Dirt procedure detected G. rostochiensis in 7 of 46 additional fields where the Method B yielded negative results, while Method B identified 2 of 46 positive fields that were negative according to Piler Dirt. When three independent samplings using Method B were summed-a rough equivalent of Method A (Canada and United States 2014) - the agreement between the methods increased to $87 \%$ for the detection of G. rostochiensis (Fig. 2B).

The number of cysts of G. rostochiensis detected by Piler Dirt and Method B in the different fields was proportional $(P<0.0001$, $R^{2}=0.885$ ) at low and moderate population densities but not similar (Fig. 3A). There was no relation at high population density (Fig. 3B). Even if the number of cysts was proportional, Method B detected significantly more cysts per kilogram of soil than Piler Dirt at low and moderate population density while the difference was not significant at higher density (Fig. 4). The above comparisons are only valid when $100 \%$ of the soil obtained by the Piler Dirt procedure is analyzed. However, in some cases, this represented a large volume of soil. When this soil was homogenized and split into subsamples of volumes equal to Method B for each strip, only a fraction of these subsamples were positive in some fields (Fig. 5A). To evaluate the Piler Dirt sample size needed to be equivalent to Method B in our dataset, we calculated the probability of a positive detection when analyzing different numbers of subsamples. For $55 \%$ of the fields, all of the subsamples were positive and only one was necessary for detection. When two subsamples were analyzed, $86 \%$ of the positive fields of our dataset were detected (with a tolerance of 5\%; that is, a $95 \%$ chance of being similar to Method B). This number increased to $90 \%$ of the fields with three subsamples and $97 \%$ of the fields with six subsamples (Fig. 5B).

\section{Discussion}

PCN are a significant threat to potato production and have important economic impacts due to yield losses but also to the expenses associated with regulatory procedures. A cumbersome step in monitoring PCN populations is field sampling. However, following the number, viability, and distribution of PCN is essential to limit its spread. In most regions, although the infested fields are regulated, potato cultivation is still possible using resistant varieties (Bélair et al. 2016). Thus, it is even more important to follow PCN populations to ensure that potato cultivars are indeed resistant and to detect the potential development of virulent populations.

In this study, we have shown that the Piler Dirt sampling technique was able to detect cysts of $G$. rostochiensis in infested fields, even after several years of nonhost crops and resistant potato cultivation. Collecting all of the soil under the machinery during potato tuber transfer to storage bins resulted in large volumes of soil. The weight of the Piler Dirt samples was, on average, 15 times higher than that of Method B and much more variable. This is not surprising because soil types can differ between the fields, as can the machinery used by growers. Also, the bioclimatic conditions at harvest were not evaluated but are expected to greatly affect the quantity of soil carried with potato tubers during harvest. This should not be problematic if a representative quantity of soil per area surveyed can be established. Because of this larger quantity of soil, analyzing all of the Piler Dirt subsamples resulted in a better detection of infested fields compared with the standard method. In our experiment, seven 
additional fields were detected by the Piler Dirt, which represents a $20 \%$ increase over the reference method. This illustrates the limitation of the reference method for the detection of infested fields with low population density.
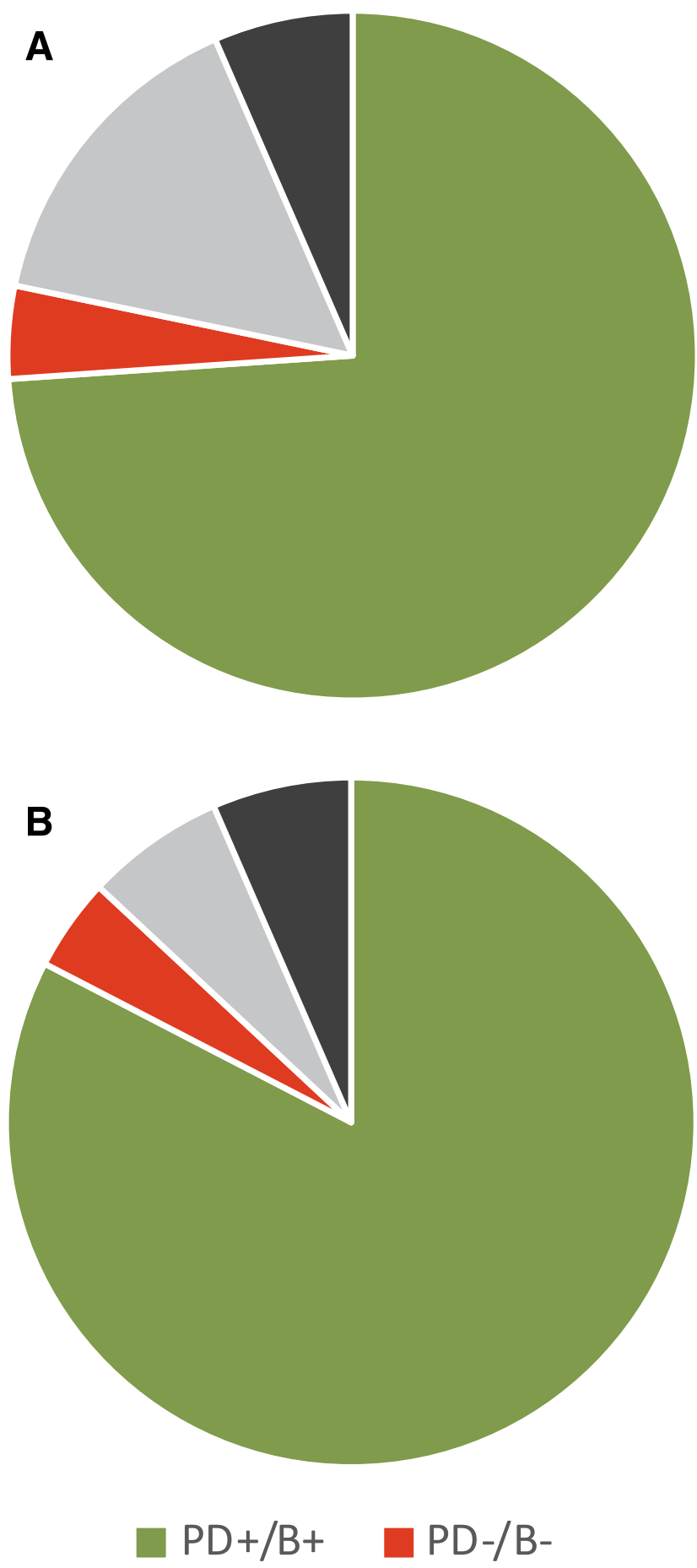

$$
\mathrm{PD}+/ \mathrm{B}-\quad \mathrm{PD}-/ \mathrm{B}+
$$

Fig. 2. Proportion of positive fields and agreement between the Piler Dirt procedure and the official standard Method B for detection of Globodera rostochiensis. A, Piler Dirt compared with Method B and B, Piler Dirt compared with the sum of three independent sampling using Method B (rough equivalent of method A). Portions in green are the fields positive with both methods, red represents the fields negative with both methods, gray represents the fields positive with Piler Dirt (PD+) but negative with Method $B(B-)$, and black represents the fields positive with Method $\mathrm{B}(\mathrm{B}+)$ but negative with Piler Dirt (PD-).
Even if the Piler Dirt technique was more sensitive, the number of cysts recovered per kilogram of soil was systematically lower, indicating a lower accuracy. However, there was a good linear relationship between Piler Dirt and Method B for quantification at low and

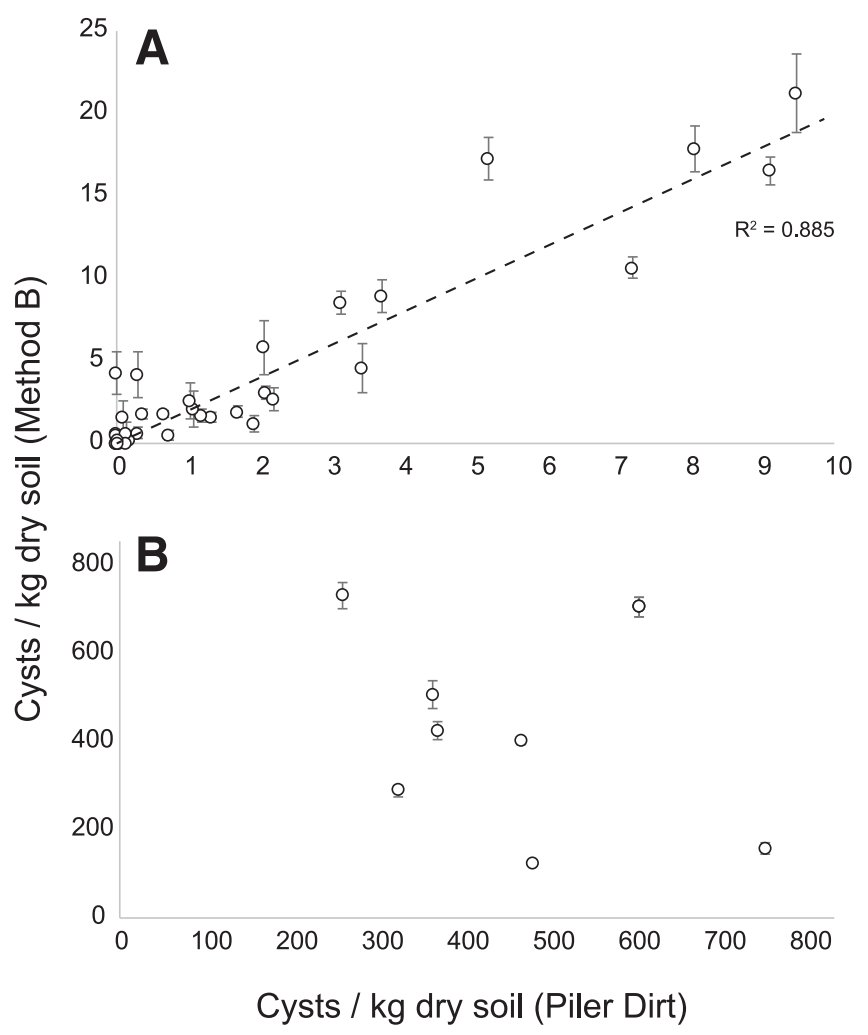

Fig. 3. Number of cysts of Globodera rostochiensis detected using the Piler Dirt procedure compared with the official standard Method B on the same area in A, fields with low to moderate initial population density and $\mathbf{B}$, fields with high initial population density. Error bars are the standard error of three independent samplings using Method B.

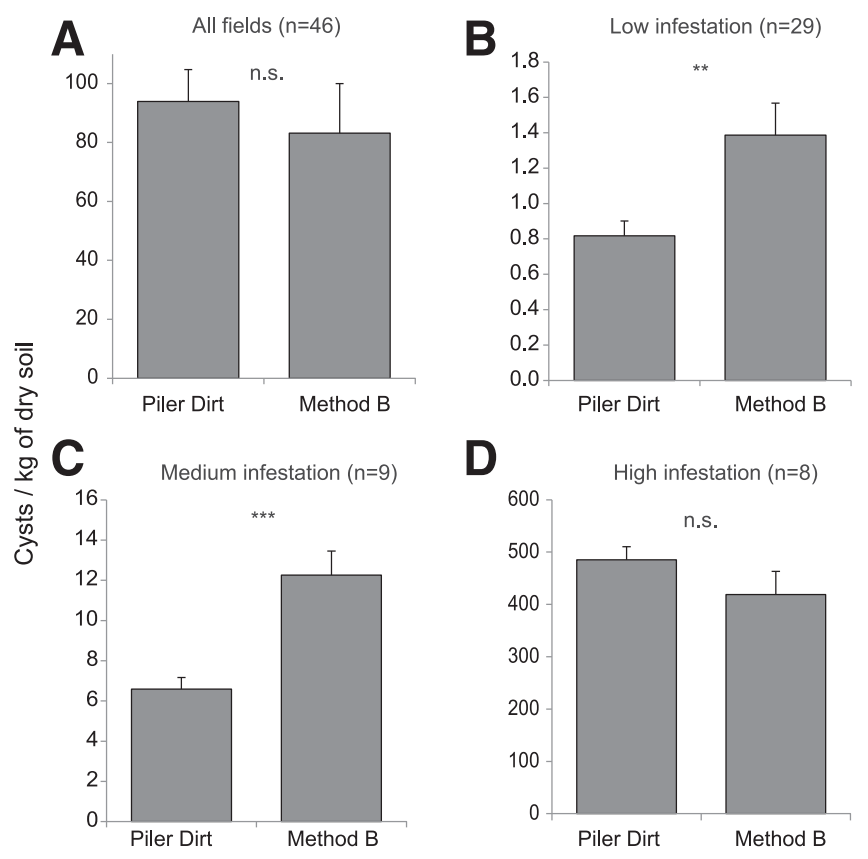

Fig. 4. Total number of cysts of Globodera rostochiensis detected using the Piler Dirt procedure compared with the official standard Method B in A, all fields; $B$, fields with low initial infestation level ( $<5$ cysts $/ \mathrm{kg}$ of dry soil); $\mathbf{C}$, moderate initial infestation level ( 5 to $25 \mathrm{cysts} / \mathrm{kg}$ ); or $\mathbf{D}$, high initial infestation level ( $\geq 100 \mathrm{cysts} / \mathrm{kg}$ ). Error bars represent the standard error for each group. 
moderate population densities. Thus, an equivalent factor could be applied. This factor was approximately 1.9 in our dataset but, without a better understanding of the causes behind this difference, it is risky to generalize the use of this multiplier. It is likely that this factor would vary greatly between production areas. Also, this relationship disappeared at higher population densities even if the overall number of cysts per kilogram of soil was not significantly different between the methods. In Europe, Goeminne et al. (2015) reported a very good relation between the number of cysts obtained by manual and tare dirt samplings after the cultivation of susceptible potato but not after resistant varieties. The authors suggested that their method probably breaks empty cysts (old cysts still present after resistant potato) that, therefore, cannot be recovered.

The main goal of this method was not to provide highly accurate quantification of the cyst number but to detect the infestation with the same or better sensitivity than the standard method. This objective was reached when the total quantity of soil was analyzed. However, this represented a lot of soil in some fields or conditions and the economy of labor during sampling was lost by the burden of soil extraction in the lab, not to mention the risks associated with the movement and disposal of these large volumes of infested soil. Thus, it is necessary to subsample the Piler Dirt samples to reduce the volume of soil while maintaining a sensitivity similar to Method B. In our dataset, six subsamples the size of a Method B sample were necessary to detect $97 \%$ of the positive fields with a tolerance of $5 \%$ (i.e., $95 \%$ chance of being similar to the reference). Because some Piler Dirt samples had less than six subsamples, applying this rule actually necessitated an average of 5.4 subsamples per strip to detect $97 \%$ of the fields $95 \%$ of the time. Performing five times more soil extractions at the diagnostic lab is a significant limitation of the method. The cost and labor time are probably still lower than scouting the field for Method B, and Piler Dirt eliminates the risk of entering the field. On the other hand, fees for shipping and disposal of contaminated waste would be increased again by five. Now, the automation of soil extraction and PCN identification steps has been
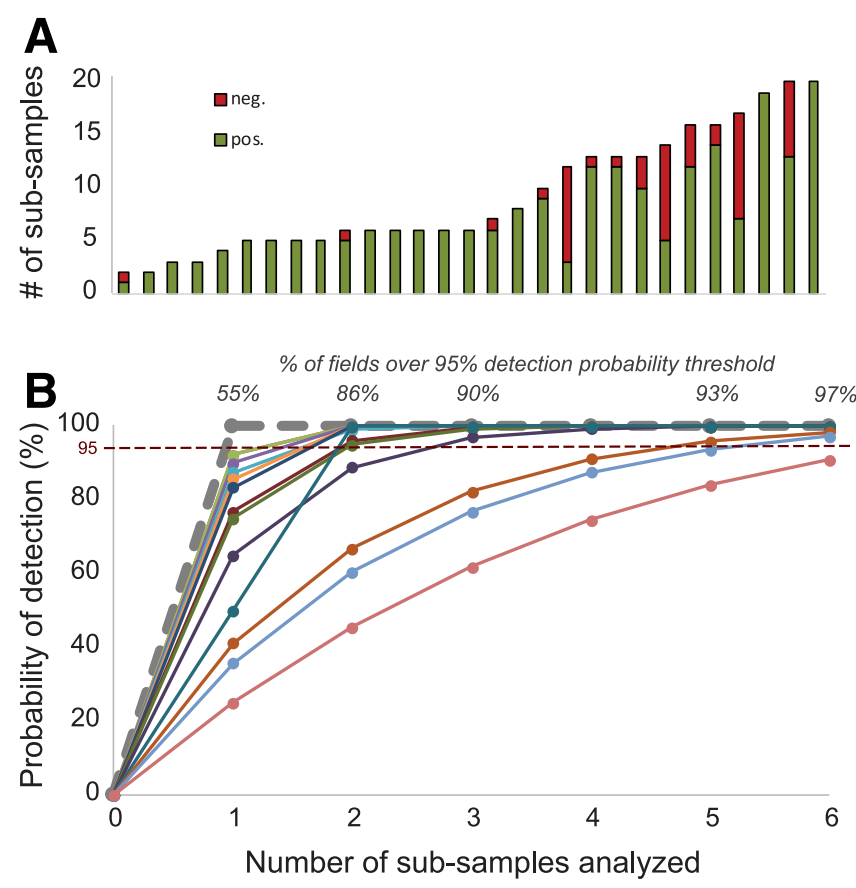

Fig. 5. Variability of Piler Dirt sampling size expressed in number of subsamples (each one equivalent to the official standard Method B) and probability of detection according to sample size A, proportion of positive subsamples by field for the detection of Globodera rostochiensis and B, number of subsamples needed to obtained a $95 \%$ probability of detection in positive fields. Only fields with positive results confirmed by the three replicates of Method B were included in the analysis. Each color represents a different field; the dashed gray line includes 16 fields for which all subsamples were positive. demonstrated by Science and Advice for Scottish Agriculture, which tests more than 20,000 soil samples each year using an automated soil-washing system and molecular detection (Reid et al. 2015). Combined with molecular quantification of egg viability (Mimee et al. 2017), this technique would cut back labor time and increase the significance of the results. Because the presence of empty cysts can persist in soil for several years, the evaluation of egg viability appears essential. Using these combined technologies could also allow coverage of an extended area and increase the chance of detection of very localized foci such as PCN escaping management on weedy hosts (Mimee et al. 2014). However, Method B remains the method of choice for the accurate quantification of cyst populations and precise localization of infestation foci in a field. The recent development of an autonomous motorized sampling device equipped with a realtime kinematic positioning system will probably remove most of the drawbacks associated with Method B and offer new possibilities. In the short term, Piler Dirt appears to be a good compromise for surveying fields for the presence or absence of PCN while reducing costs for the regulatory agencies. However, the implementation of the method will be challenging. The Piler Dirt samples have to be collected during harvest. Because many fields are harvested simultaneously in different regions, it would be logistically impossible to dedicate a team for that. Also, the sampling would require the participation of farmers at a time when they are already overwhelmed and stressed. In contrast, Method B can be realized at any time following harvest or even during nonhost years.

Quarantine procedures and a careful monitoring of $\mathrm{PCN}$ populations have shown to be effective to contain this threat but we must remain vigilant because new infestations or the development of virulent populations are possible, especially in light of climate change and global trade that accelerate the development of nematodes and increase the chances of new introductions (Gendron St-Marseille et al. 2019; Mimee et al. 2015a).

\section{Acknowledgments}

We thank the CFIA staff from St-Hyacinthe, QC and Charlottetown, PEI for their support in sampling, soil extraction, PCN identification, and quantification and two anonymous reviewers for their suggestions and very useful comments.

\section{Literature Cited}

Baldwin, G. J., and Mundo-Ocampo, M. 1991. Heteroderinae, cyst-and non-cystforming nematodes. Pages 275-362 in: Manual of Agricultural Nematology. R. W. Nikle, ed. Marcel Dekker Inc., New York, NY.

Bélair, G., Dauphinais, N., and Mimee, B. 2016. Evaluation of cultural methods for the management of the golden nematode (Globodera rostochiensis) in Quebec, Canada. Can. J. Plant Pathol. 38:209-217.

Boucher, A. C., Mimee, B., Montarry, J., Bardou-Valette, S., Bélair, G., Moffett, P., and Grenier, E. 2013. Genetic diversity of the golden potato cyst nematode Globodera rostochiensis and determination of the origin of populations in Quebec, Canada. Mol. Phylogenet. Evol. 69:75-82.

Brodie, B. B., Evans, K., and Franco, J. 1998. Nematode parasites of potato. Pages 87-132 in: Plant Parasitic Nematodes in Temperate Agriculture. K. Evans, D. L. Trudgill, and J. M. Webster, eds. CAB International, Wallingford, U.K.

Canada and United States. 2014. Guidelines on surveillance and phytosanitary actions for the potato cyst nematodes Globodera rostochiensis and Globodera pallida. https://www.aphis.usda.gov/plant_health/plant_pest_info/nematode/ downloads/potato_guidelines.pdf

CFIA. 2006. Golden nematode infested places order. Canadian Food Inspection Agency. http://www.inspection.gc.ca/plants/plant-pests-invasive-species/nematodesother/golden-nematode/order/eng/1337006958263/1337007108429

Duceppe, M.-O., Lafond-Lapalme, J., Palomares-Rius, J. E., Sabeh, M., Blok, V., Moffett, P., and Mimee, B. 2017. Analysis of survival and hatching transcriptomes from potato cyst nematodes, Globodera rostochiensis and $G$ pallida. Sci. Rep. 7: Article 3882.

FAO. 2018. FAOSTAT World production of agricultural commodities for 2016. Food and Agriculture Organization of the United Nations. www.fao.org/faostat/

Fenwick, D. W. 1940. Methods for the recovery and counting of cysts of Heterodera schachtii from soil. J. Helminthol. 18:155-172.

Gendron St-Marseille, A.-F., Bourgeois, G., Brodeur, J., and Mimee, B. 2019 Simulating the impacts of climate change on soybean cyst nematode and the distribution of soybean. Agric. For. Meteorol. 264:178-187.

Goeminne, M., Demeulemeester, K., Lanterbecq, D., Proft, M. D., and Viaene, N. 2015. Detection of field infestations of potato cyst nematodes (PCN) by sampling soil from harvested potatoes. Asp. Appl. Biol. 130:105-110.

Goeminne, M., Demeulemeester, K., and Viaene, N. 2011. A method for estimating the contribution of seed potatoes, machinery and soil tare in field 
infestations with potato cyst nematodes on a national scale. Commun. Agric. Appl. Biol. Sci. 76:311-318.

Hafez, S. L., Sundararaj, P., Handoo, Z. A., Skantar, A. M., Carta, L. K., and Chitwood, D. J. 2007. First report of the pale cyst nematode, Globodera pallida, in the United States. Plant Dis. 91:325.

Mahran, A., Turner, S., Martin, T., Yu, Q., Miller, S., and Sun, F. 2010. The golden potato cyst nematode Globodera rostochiensis pathotype Ro1 in the Saint-Amable regulated area in Quebec, Canada. Plant Dis. 94:1510.

Mimee, B., Andersen, R., Bélair, G., Vanasse, A., and Rott, M. 2014. Impact of quarantine procedures on weed biodiversity and abundance: Implications for the management of the golden potato cyst nematode, Globodera rostochiensis. Crop Prot. 55:21-27.

Mimee, B., Dauphinais, N., and Bélair, G. 2015a. Life cycle of the golden cyst nematode, Globodera rostochiensis, in Quebec, Canada. J. Nematol. 47: 290-295.

Mimee, B., Duceppe, M.-O., Véronneau, P.-Y., Lafond-Lapalme, J., Jean, M., Belzile, F., and Bélair, G. 2015b. A new method for studying population genetics of cyst nematodes based on Pool-Seq and genome-wide allele frequency analysis. Mol. Ecol. Resour. 15:1356-1365.
Mimee, B., Soufiane, B., Dauphinais, N., and Bélair, G. 2017. A qRT-PCR method to evaluate viability of potato cyst nematode (Globodera spp.). Can. J. Plant Pathol. 39:503-513.

Phillips, M. S. 1994. Inheritance of resistance to nematodes. Pages 319-338 in Potato Genetics. J. E. Bradshaw and G. R. Mackay, eds. CAB International, Wallingford, U.K.

Reid, A., Evans, F., Mulholland, V., Cole, Y., and Pickup, J. 2015. Highthroughput diagnosis of potato cyst nematodes in soil samples. Pages 137-148 in: Plant Pathology, Techniques and Protocols. C. Lacomme, ed. Humana Press, Springer, New York, NY, U.S.A.

Sabeh, M., Duceppe, M.-O., St-Arnaud, M., and Mimee, B. 2018. Transcriptomewide selection of a reliable set of reference genes for gene expression studies in potato cyst nematodes (Globodera spp.). PLoS One 13:e0193840.

Seehofer, H. 2007. Council Directive 2007/33/EC of 11 June 2007 on the control of potato cyst nematodes and repealing Directive 69/465/EEC. Off. J. Eur. Union 156:12-22.

Sun, F., Miller, S., Wood, S., and Côté, M. J. 2007. Occurrence of potato cyst nematode, Globodera rostochiensis, on potato in the St-Amable region, Quebec, Canada. Plant Dis. 91:908.

Zaheer, K., and Akhtar, M. H. 2016. Potato production, usage, and nutrition-A review. Crit. Rev. Food Sci. Nutr. 56:711-721. 